\title{
A Bayesian Approach for User Modeling in Dialogue Systems
}

\author{
AKIBA, Tomoyosi and TANAKA, Hozumi \\ Department of Computer Science \\ Tokyo Institute of Technology \\ 2-12-1 Ôokayama Meguro Tokyo 152 Japan \\ \{akiba, tanaka\}@cs.titech.ac.jp
}

\begin{abstract}
User modeling is an important components of dialog systems. Most previous approaches are rule-based methods. In this paper, we propose to represent user models through Bayesian networks. Some advantages of the Bayesian approach over the rulc-based approach are as follows. First, rules for npdating user models are not necessary becanse updating is directly performed by the evaluation of the network hased on probability theory; this provides us a more formal way of dealing with uncertainties. Second, the Bayesian network provides more detailed information of users' knowledge, because the degree of belief on each concept is provided in terms of probability. We prove these advantages through a preliminary expcriment.
\end{abstract}

\section{Introduction}

Recently many researchers have pointed out that user modeling is important in the study of dialog systems. User modeling does not just render a dialog system more cooperative, but constitutes an inclispensable prerecuisite for any ftexible dialog in a wider domain[9]. The nser models interact closely with all other components of the system and often camot easily be separated from them. For example, the input, analysis component, refers to the nser's knowledge to solve referential ambiguities, and the output generation component does the same for lexical choices.

The concepts are usually explained by showing their relations to the other known concepts. Thus, for the dialog system it is important to guess what the user knows (user's knowledge) in order to explain new concepts in terms of known concepts. For example, consider that the system explains the location of a restanrant to the user. It might be useless to tell the user the position in terms of the absolute coordinate system, since the user's mental model is not based on the absolute coordinate. Therefore, the system should show the relative location from the location the user already knows. It is difficult to predict which locations the nser, who perhaps is a stranger to the system, knows. Though the systen could attempt to acquire the information by asking the user about her knowledge, too many questions may irritate the user. Such a system is considered medhanical and not leelpful. Therefore, the systicm is required to guess the user's knowledge by finding clues in the user's utterance and to refine the user's model incrementally.

In the ner modeling component of UC[5], scveral stereotyped user models which vary the user's level of expertise were prepared beforehand and the appropriate model was selected based on the user's utterances. In the approach used by Wallis and Shortliffe [12], the expertise level was assigned to all concepts in the user model. The system guessed the user's level, and the concepts with the expertise level lower than her level are considered to be known by her. This model can deal with the level of expertise more appropriately than UC, because the system does not have to prepare the multiple user models for each expertise level.

The approach of preparing several nser models and adopting one, however, is an approximation of user modeling. The expertise level of the user is continuous and, in general, the unique measurement of expertise level is not appropriate for some domains, specifically the domain of town guidance considered in this paper, because the areas that are known differ with the users.

Another problen of user modeling is updating the model as the dialog progresses. At the beginning of the dialogue the system cannot expect the user model to be accurate. As the dialogue progresses the system can acquire clues of the nser's knowledge from his utterances. Also, the system can assume that the concepts mentioned are known to the user. Thus, updating the user model should be performed incrementally.

One elifficulty of updating user models is dealing with nncertainties. The clues that can be obtained from the user's utterauces are uncertain, the information may conflict with what las been obtained. and, as a result, the user model inay be revised. The effects of the system's explanation are also uncertain. Furthermore, reasoning about the user's knowledge must be performed on the basis of uncertainties. Most previous approaches to this problem are rule-based methods. Cawsey [2] sorted the update rules in order of their reliability and applied them in this order. In another approach, the mechanism such as 'TMS[6] or nonmonotonic logic[1], is used to maintain the consistency of 
the model. It seems that rule-based approaches have a polential defect for dealing with uncertainties[4]. 'Tho Baycesian approach can deal with both ancertain (ambignous) cvidences and uncertain reasoning straight-forwardly.

In this paper, we propose a probabilistic: approach for user modeling in dialog systems. The Bayestan networks are used to represent the user's knowledge and draw inferences from that, and provide the fine-grained solutions to the problens previously mentioned. In spite of the potential advantage of the Bayesian ap. proach, there are fow athenpts to employ it in user modeling.

The arlvantages of the Bayesian approach oven the rule-based approach are as follows. First, rules for uprlating user models are not necessary. Cawsey [2] pointed out there are four main sources of information that can be used to nprate the nser model what tho usce says and asks, what the system tells the user, the level of expertise of the neser, and relationships between conceptis in the domain. They can be incorporated in the representalion of 13ayesian networks and cant be used to npelate the user model by evaluating the networks.

Second, the Bayesian nelwork provides more detailed information of users' knowledge. Tn the case of binary modeling of knowledge, whereby either the aser knows or does not know a conceple, it is too coarse to judge the model under uncet lainty. Therefore, usially, the degree of beliel is assigned to all concepts in the model. It is not clear where the degree of belief comes from or what it means. On the other hathd, however, the Bayestan approach provides lite degree of belief for cloar sonantics, which is probability.

The remander of this paper is organized in four sections. Section 2 is devoted to an outline ol Bayesian networks. Section 3, knowledge representation in termis of Bayesian networks is discussed. If the nodel is once repesented, then the rpdating of the model will be taken care of through the evaluation of the network. Section 4, some examples are given along with an experiment to show the arlvantage of our approach. Section 5 concludes this paper.

\section{Bayesian Networks}

Reasoning based on probability theory rexpires probabilistic: models to be specified. In general, a complete probabilistic model is specified by the joint probabilities of all randon variables in the domain. 'The problem is that the complete specification of the joint probabilities recuires absurd announts of numbers. lior example, consider the case where all random variables are binary, having a value 0 or 1 , the connplete probabilistic model is specified by $2^{n}-1$ joint probabilities. (Assuming $n$ binary ranclom variables, $x_{1}, x_{2}, \ldots x_{n}$, the distribution is specified by the probabilitics, $P\left(x_{1}=0, x_{2}=0, \ldots, x_{n}=0\right), P\left(x_{1}=1, x_{2}=\right.$ : $\left.0, \ldots, x_{n}=0\right), \ldots, P\left(x_{1}=1, x_{2}=1, \ldots, x_{n}=1\right)$, that sum 11p to unity so one of them can be automatically gained.) Moreover, in practice it is difficult to explicitly specify the joint probability. Concerning our purpose of modeling the user's knowledge, where a random variable corresponds to a concept and whose value corresponds to the user's knowledge of the concept, it is almost impossible to specify all joint probabili. ties because this involves enumerating all of the user's knowledge patterns.

Bayesian networks need far fower probabilities and cau provide the complete probabilistic models. The information that componsates for the gap) is qualitative, which is obtained by investigating the nature of the domain. The Bayesian network has both qualilative and quantitative characteristics, therefore, we can represent, the knowledge qualitatively and reason about probability quantitatively. Formally, Bayesian networks are directed acyclie graphs (D $\Lambda \mathrm{G}$ ) with the nodes representing at randon variable and the directed ares representing the dired dependent relation bet.ween the linked variables. If a are goes from one node to another, we saly that the former is a parent node of the latter, and the latter is a child of the formes. The distribution on the network is specified to all nodes $x$ its probability $P(x \mid p(x))$ conditioned by the set of it.s parent nodes $p(x)$. The nodes without parents are assigned the prior probabilities $P^{\prime}(x)$. That is all that is necossary for specifying a conplete probabilistic nodel [10].

the roasoning on Bayesian networks corresponds to evaluating the posterior probability $P(x \mid l)$ on all nodes $x$ given the evidence $r$ that is specifed hy providing certain values to a certain subset of nodes in the networks (for instance, $z:=\{y=1, z=0\}$ for some nodes $y$ and $z$ ). The evaluation of the network is doue in general by the stochastic sinulation [10]. The uprating of the user models are directly performed by evaluating the network once the knowleclge of the doInain has been correctly represented by the Bayesian. network. In the next section, we discuss knowledge representation with Bayesian notworks.

\section{Knowledge Representation with Bayesian Networks}

\subsection{Designing the Language}

We have sairl the nodes in the Bayesian notwork are random variables that range over some values. In order to represent knowledge in terns of the Bayesian network, we must design the language for the sentences assigned to the nodes of the network. We first assume that the variables have two possible values, so that the sentences lave truth values, that is, 1 (true) or 0 (false). Note that this assumption is not crucial; we may assign values such as KNOWN, NOT-KNOW, NO-[NJORMATION as in UMl' [11]. 
The type of sentences may depend on the application we pursue. For general explanation, it is important to make a clear distinction between the two user's states; knowing the name of a concept and knowing the other attribute of the concept. For exanple, suppose the aser asked the following:

"Where is FRISCO ?"

where FRISCO is the name of a record store. From this question, the system infers that the user knows the name of the store, but does not know its location.

Now we will give a precise definition of our language. All the sentences in the language have the form

\section{〈label $\rangle:\langle$ content $\rangle$}

where (label) is one of PRE, POST, JUDGE, TOLD, and TELL, and (content) is represented by a term of the first-order predicate logic. An object and an expertise field are represented by an atomic symbol, and an attribute of an object is represented by a function symbol. For example, store001(object), records_collector(expertise ficld), location(store001) (attribute), and so forth.

The user's knowledge about an attribute is represented by five sentences, all liaving the same (content) representing the attribute, and one of the five labels. The sentences labeled PRE, express that the user knows the attribute prior to the dialogue session, while those labeled POST, express that the user has come to know it during the session. For instance, PRE: location(store001) means that the user have already knows the location of store001 before the interaction starts, while POST: location(store001) means the user has come to know the location through the system's explanation. The sentences labeled JUDGE, express the user's current knowledge and is used to exploit the user model by other components in the dialogue system. For instance, JUDGE: location(store001) means the user now knows the location of store001. The sentences labeled TOLD and TELL, express the evidence, gained by the user's utterance and the system's explanation. For instance, TOLD: name(store001) means the nser has indicated by the clues that she knows the name of store001, while TELL: name(store001) means the system has explained the nane. For exception, in the case of location, the form TELL: location(X)(where $\mathrm{X}$ is some object ID) is not used becalse a location is explained in terms of the relative location of another object. Instead, the form TELL: relation(X, $\mathrm{Y})$ (where $\mathrm{X}$ and $\mathrm{Y}$ are some object IDs) is nsed.

The sentences representing objects and expertise ficlds have only the label PRE. The sentence representing an object (e.g. PRE: storo001) means that the user knows the object, that is she knows most of the attributes of the object. The sentence representing an expertise fiell (c.g. PRE: records_collector) means that the user is an expert of the field, that is she knows the olsjects related to the expertise ficld.

\subsection{Constructing the Networks}

As mentioned, ares of the Bayesian network represent direct probablistic influence between linked variables. The directionality of the arcs is essential for representing nontransitive dependencies. In order to represent the knowledge in terms of Bayesian Network, we must interpret the qualitative relation between the sentences that are represented by our language as a directed are or some such combination of ares.

In our case, the network has two sub-uetworks. One represents the user's knowledge before the dialog session, which is used to guess the user's model from her utterances. The sentences assigned to the nodes in this part have either the label PRE or TOLD. We call this subnetwork the prior part. The other subnetwork in which the nodes have either the label POST or TELL is used to deal with the influence of the system's utterances. This subnetwork we call the posterior part. It is important to make a clear distinction. Considering that the system explains a concept, it is not proper to assume that the user knows some other related conecpts. For example, if the user utters that she knows some location $x$ then it can be inferred that she also knows locations that are close to $x$. But that is not true if the location $x$ is explained by the system.

The relations in the prior part of the network are categorized into four types as follows:

(1) the relations between objects in an expertise field

(2) the relations between attributes of ohjects

(3) the relations between an object and its attributes

(4) the relations between an attribute of an object and the evidence that the user knows it

The relations (1) are concened with the expertise field. The objects in the same expertise field are related through the expertise field node. We introduce the ares that go from the expertise field node to the object nodes belonging to that fiekl. For example, ares go from the node of "records collector" to that of "Compact Disk", "Tower Records" (name of a record store) and so on. The level of expertise can be controlled by the conditional probabilities assigned to the object nodes conditioned by the expertise field node. In this framework, we can introduce arbitrary numbers of expertise fields, all of which can be assigned the level of expertise.

The relations (2) are concerned with the domain knowledge. In our domain, those are the relations between the locations, which are based on the assumption that the user probably knows the locations close to the location she knows. The relations are assumed to be symmetric. A single directed are of Bayesian networks does not represent i symmetric relation. In order to represent a symmetric relation, we introduce a dummy evidence node, whereby two ares go forth from the two location nodes as shown in figure 1. The prior 


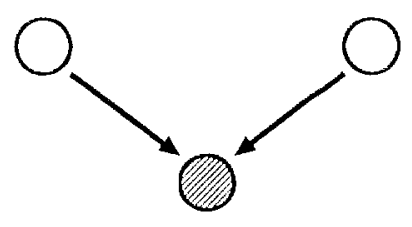

dummy node

ligure 1: Symmetric relation

conclitional probabilities of the dunmy node have high value if the two parent nodes have the same value.

The relations (3) are concerned wilh general knowledge, such as knowing an object well implicates know. ing its attributes. In order to represent such kind of relations, we introduce the ares to go from the node of an object to the nodes of its attributes.

The are corresponding to the relation (4) is introduced to go from the node of an attribute of an object to an evidence node. The attribute node and the evidence node have the same content, while they have the different labels, PRE and TOLD.

In the posterior part of the network, there are only arcs representing the relations (4). The attribute nodes and the evidence nodes are labeled I'OST and TELL. In anddition, the TELL node may lave more lian one parent, node becanse the explanations of the attribute are made by referring to the other attributes. Actually, in our town guidance domain, the system explains the now location using the locations that the nser alrealy knows. For instance, the nodes POST: location(storo001) and POST: location(store002) are parcuts of the node TELL: relation(store001, store002) when the system explain the location of store001 by using the location of store002. The more the system shows the relations, the deeper the user's understanding becones.

The ambiguous evicience can be dealt with straightforwardly in the Bayesian approakh. An evidence node can have more than one parent node to represent the ambiguity. For example, when dealing with spoken inputs, it might be ambiguous that the user said either "tower records" or "power records." If both record stores exist, au evidence node labeled TOLD is introduced as a child node for both nodes, PRE: name(tower) and PRE: name(power) (figure 2).

Finally, we introduce the ares that comect the two subnetworks. For each attribute, there are three kinds of nodes labeled PRE, POST, and JUDGE. 'J'he two are are drawn from the PRE node to the JUDGE node and the POST node to the JUDGE node. That means the user knows the attribute cither because he already knew it before the current, dialogue session or becanse it has been explained by the system during the session.

The example of the resulting network is shown in figure 3.

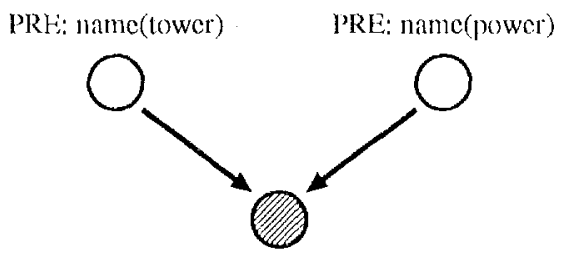

TOI.l): name(?ower)

Figure 2: Anbiguous evidence

\section{Examples}

Suppose the user asks the system to show the way to a record store named FRISCO in a town (figure 4). The system uses the network in figure 3. The dialogne starts with the user's request.

\section{(1) nser: Where is FRISCO?}

In practise, the input analysis component is needed to obtain evidences of the uetwork from the usser's utterances, but this proecss is beyond the scope of this paper. $3 y$ malyring the inpont, the system obtains the information that the nser knows the name of a certain store, but does not know its location. The inpute, i.e. the evidence, to the network is $l_{f}=\{$ TOLD: name(frisco) $=$ 1, TOLD: location (frisco) $=0\}$. Fvaluating the degree of belief of each concept $x$ by using the posterion probability $P(a \mid$ TOLD: name(frisco) $=1$, TOLD: location(frisco) $=0$ ) gives the resulting user model. Though this result can be directly obtained by evalu. ating the network, we will briefly trace onr reasoning for explanatory purposes. (Note that the actual process is not easy to explain as all nodes of the network influence each other, that is the reason why sinntation is needed for evaluation.)

The user knows the nane FRISCO, which represents that she las the high expertise level for records collectors and raises the probability of the node PRE: records_collector and also raises that of the node of other record stores, Tower Records(PRE: tower), Wave Records(IRE: wave). 'These nodes then affect the node of their attributies, PRE: location(tower), PRS: name(tower), PIRE: location(wave), and so on. That raises the probability of the location node UANDS Departmont (PRE: location(hands)), which is close to the location the ner (probably) kuows, i.e. PRE: location(wave).

Next, the system generates the answer by asing the resulting user model. This task is done by a planner for utterance generation. 'The system may decide to use the location of II $A$ NDS.

(2) system: It is $300 \mathrm{~m}$ to the south from TLANDS Departinent. 


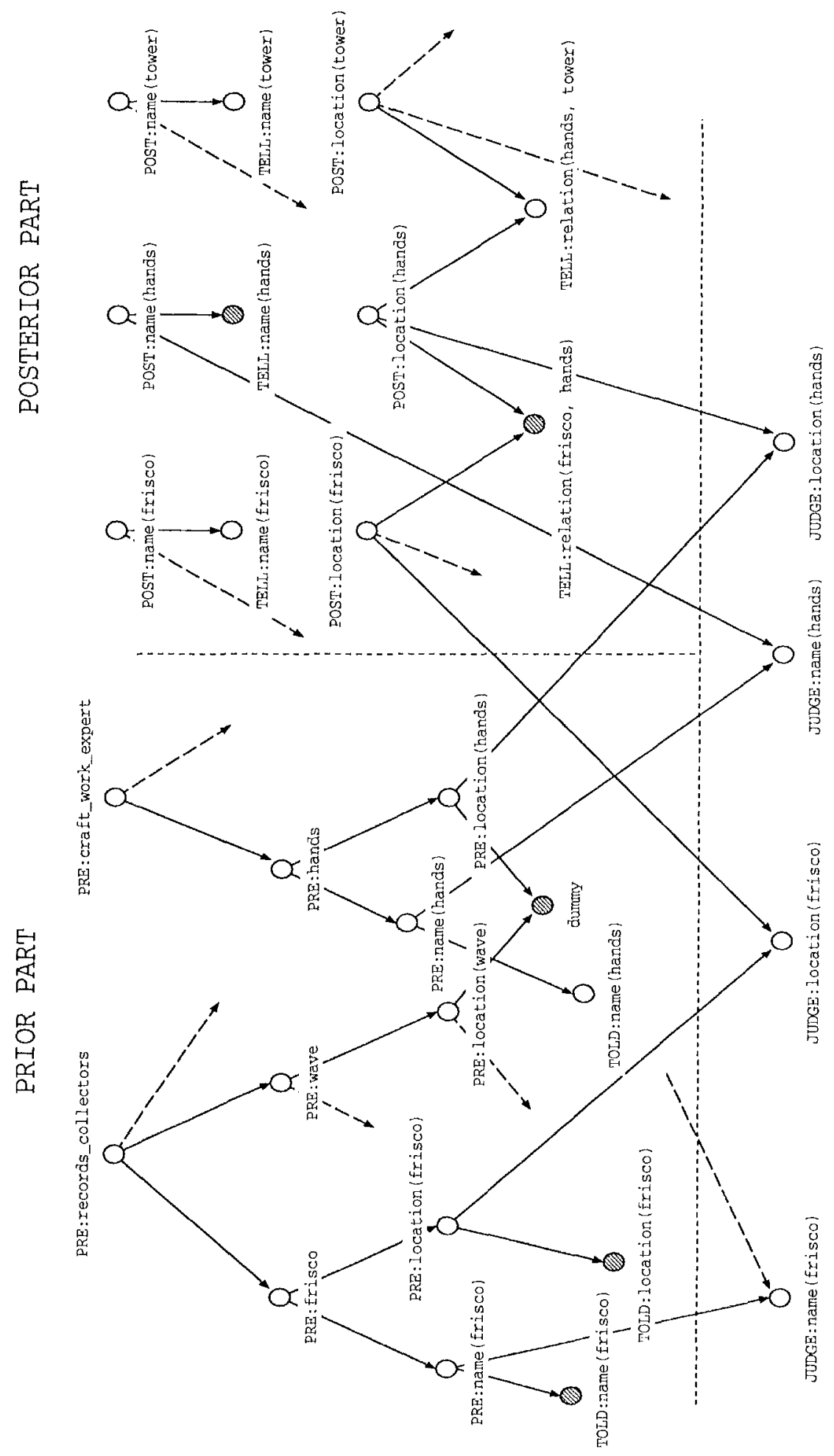

Figure 3: Example of a network 


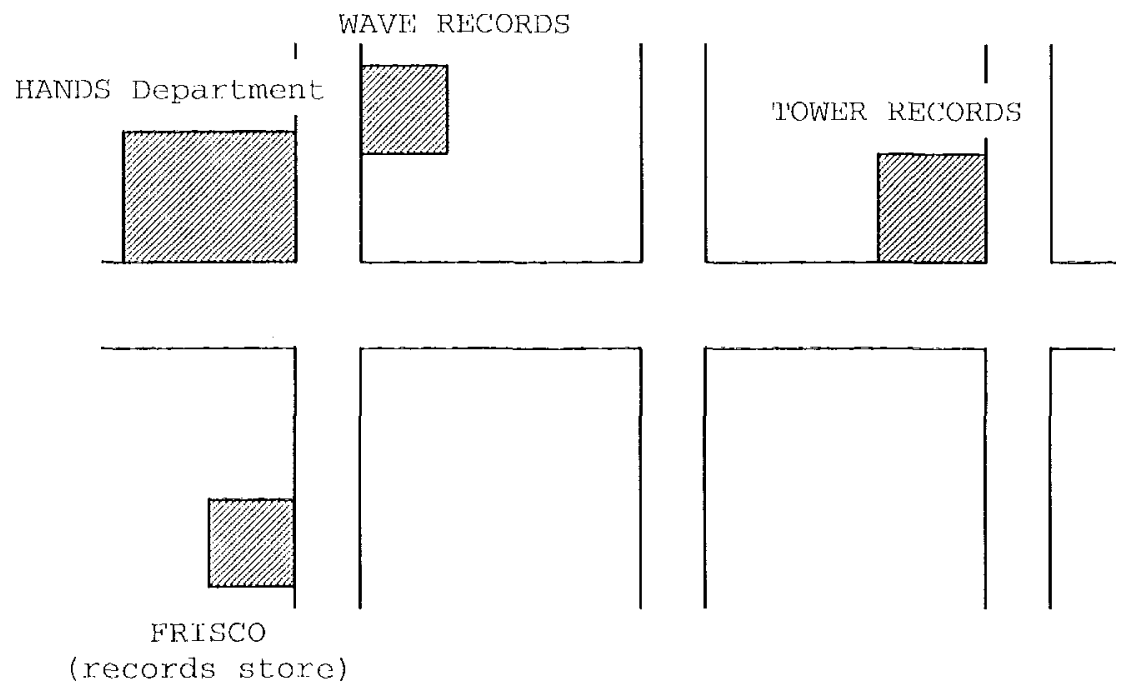

Figure $4: \wedge$ map of a town

Alter uttering the sentence, the system ands the cvidence, TELL: name(hands) - - 1, TELL: relation(hands, frisco) $=1$, to the network. Noto that the explanation of the location is made by show. ing its relation to ofluer locations. That makes tho probability of the node POST: location(frisco), P(POST: location(frisco)| $t)$ raise, where $b$ represents all evidence olstained. 'The next utilerance of the user is:

(3) user: I don't know whore ILANDS is.

This input gives the system the evidence, TOT, location(hands) $=0$. After obtaining this evidence, the beliof is revised. The probability of the node PRE: location(hands) falls, which in turn canses the probability of the node PRH: location(wave) to fall.

Next, the planer may try to explain the localion of II $\Lambda$ NI)S, by using the location of Tower Recordis which gives the evidence TELIs: relation(hands, tower) -1.

(4) system: IJANDS is two blocks away to the west from tower Records.

'This explanation not only can influence the user's understanding of the location of HANDS but also the location of FRISCO, because the evidence raises the posterior probability of the node POS'T: location(frisco) through the node POST: loca tion(hands).

Fvaluation resulds of the above dialogue are shown in Table 1.

\section{Conclusion}

We have proposed the Bayesian approach for aser modeling in dialogue systems. The knowledge representation, in terms of Bayesian networks, has been disceussed. Reasoning would be antomatically and directly performed by evaluating the network followed by stochastic sinulation.

Most exact solutions for the interesting problens in artificial intellipence are known to have NP-harel computational complexity. 'J'hus, it lats been recognized that solving them by an approximate method is a more realistic approad. The Bayesian networks are evaluated by the stochastic simulation, which is the approxinate solution of probabilistic reasoning. The simulation cost, lowever, is still expensive with the present computing resources. 'The parallel implementation las reported good performance results [7].

After gaining the accurate expectalinus of nser nodels, a mechanism to use them for utterance generation is required. 'T'lis will be done by planners for ulterance generation, which try to achieve the system's goals. The probabilities in the user model contribute to measure to what extent the plan will succeed.

In the study of natural language processing, Bayesian approaches have been adopted in the field of plan recognition [3] and lexical disanbiguation [7]. We have adopted the Jayesian networks for user nodeling becanse we have perceived that user modeling is one of the core components of dialogue systems whose behavior strongly influences the other parts of the system. Wo endeavor to construct the experimental dialogue system that accepts the users' inputs by speech recognition[8]. Starting with nser modeling, we will ex- 


\begin{tabular}{|l||c|c|c|c|c|}
\hline \multirow{2}{*}{ node } & \multirow{2}{*}{ prior } & \multicolumn{4}{|c|}{ probabilitics after } \\
the utterance (n)
\end{tabular}

Table 1: The zesult of evaluation

pand the adoption of Bayesian approaches in most of the components in the system. The approaches must be quite effective in the other components, and lead to a system whose components closely interact with each other on the common basis of probability theory.

\section{References}

[1] Douglas E. Appelt and Kurt Konolige. A nonmonotonic logic for reasoning about speech acts and belief revision. In International Workshop on Nonmonotonic Reasoning, pp. 164-175, 1988.

[2] A. Cawscy. Explanation and Interaction. MIT Press, 1993.

[3] E. Charniak and R.P. Goldman. A bayesian model of plan recognition. Artificial Intelligene, Vol. 64, No. 1, pp. 53--79, 1983.

[4] Peter Cheeseman. In defence of probability. In the Proceedings of the Intermational Joint Conference on Artficial Intelligence, p1. 1002-1009, 1985.

[5] David N. Chin. KNOME: Modeling what the user knows in UC. In $A$. Kobsa and W. Wallster, editors, User Models in Dialog Systems, chapter 4 , pp. 74-107. Springer-Verlag, 1989.

[6] J. Doyle. A truth maintenance system. Artificial Intelligence, Vol. 12, pp. 231 272, 1979.

[7] Iscila M. R. Ei\%irik, Valmir C. Babosa, and Sucli B. T. Mendes. A bayesian-network approacl to lexical disambiguation. Cognitive Science, Vol. 17, pp. 257--283, 1993.

[8] K. Itou, S. Hayamizu, and H. Tanaka. Continuous speecl recognition by context-dependent phonetic HMM and an efficient algorithm for finding n-best. sentence hypotheses. In In Proceedings of International Coference on Acoustics, Speech, and Signal Processing, 1992.
[9] A. Kobsa and W. Wahlster, editors. User Models in Dialog System.s. Springer Verlag, 1989.

[10] J. Pearl. Probabilistic Reasoning in Intelligent Systems. Morgan Kaufmann, 1988.

[11] J. Slecman. UMFE: $\Lambda$ user modelling front end subsystem. International Journal of ManMachine Studies, Vol. 23, pp. 71-88, 1985.

[12] J.W. Wallis and E. H. Shortliffe. Customized explanations using causal knowledge. In B.G. Buchanan and E.II. Shortliffe, editors, Rule Bused Expert Systems: The MYCIN experiments of the Stanford Heuristic Programming Project, 1). 371 390. Addison Wesley, 1985. 


\section{Reserve Papers}


